\title{
Experimental modules covering imaging, diffraction, Fourier optics and polarization based on a liquid- crystal cell SLM
}

\section{Andreas Hermerschmidt}

Andreas Hermerschmidt, "Experimental modules covering imaging, diffraction, Fourier optics and polarization based on a liquid-crystal cell SLM," Proc. SPIE 9666, 11th Education and Training in Optics and Photonics Conference, 96660Q (5 June 2009); doi: 10.1117/12.2207974

EDIE Event: Eleventh International Topical Meeting on Education and Training in Optics and Photonics, 2009, St. Asaph, United Kingdom 


\title{
Experimental modules covering imaging, diffraction, Fourier optics and polarization based on a liquid-crystal cell SLM
}

\author{
Andreas Hermerschmidt, Holoeye Photonics AG, Albert-Einstein-Str. 14, 12489 Berlin, \\ Germany
}

\begin{abstract}
In close collaboration with four German universities, we have developed tutorials for experiments based on a transmissive liquid-crystal spatial light modulator (SLM). The experimental tutorials are grouped in six project modules, which cover a wide range of phenomena and have different levels of difficulty. At a basic level, students can investigate the SLM in its probably most well-known application as an image-generating element in a simple optical projector setup. At more advanced levels, the application as an adaptive optical element can be investigated in three different projects covering wave-optical phenomena. The fields covered include Fourier Optics using the SLM as a dynamic fan-out beam-splitter or kinoform, Computer-Generated Holography and basic Interferometry. For the support of these projects, software was developed which permits the generation of adaptive optical structures by the student with a user-friendly interface, while the underlying algorithms are explained in the theoretical tutorial. The modulation of the light by the twistednematic liquid crystal cells of the SLM can be investigated in the two most advanced projects. In the first one, the parameters of the cell and the components of its Jones matrix can be derived from transmission measurements with rotatable polarizers at a number of different wavelengths. This project gives insight to the Jones matrix calculus at the level required for the analysis. In the second one, the complex-valued transmission of the SLM is determined by measuring the diffraction efficiency of dynamically addressed Ronchi gratings.
\end{abstract}

Keywords: Spatial Light Modulator, Fourier Optics, Diffraction, Polarization

\section{INTRODUCTION}

Liquid crystal (LC) based micro-displays by now have been used for the manipulation of incident light waves for almost two decades. Initially components taken from commercial projectors were used to explore the suitability of such micro-displays for phase modulation, in order to use them as adaptive optical components like e.g. Fresnel lenses, Diffractive Beam-Splitters or aberration compensators. Since then, new dedicated devices referred to as Spatial Light Modulators (SLMs) have emerged. There are numerous experimental applications for such adaptive optical devices; however it is also evident that SLMs are quite suitable for optics education because especially the fields of Diffractive Optics and Fourier Optics are much more accessible for student laboratories with such flexible dynamic devices, compared to their static counterparts.

Therefore, the development of the 'OptiXplorer' education kit based on an 'LC2002' SLM started in our company several years ago, in close cooperation with a few German universities. Because an SLM is a flexible device, the laboratory tutorials which where developed in this way have quite different topics in optics, so we decided to group the different experiments into modules. While one group of modules is dedicated to the application side and uses the SLM as an amplitude modulator (i.e. an image source) or as a phase modulator, another group of modules is dedicated to the characterization of the device, which is partly due to the history of SLM development.

Because the main purpose of the LC based micro-displays was to be used as an image source for projection applications, not all parameters that are required for other applications - like phase modulation - were given on the datasheets of the manufacturers. The derivation of those missing parameters from experiments was a relevant topic, and indeed some properties of the liquid crystal cells can be obtained from rather simple 
experimental set-ups. Therefore these experiments were developed into independent modules of the 'OptiXplorer' education kit which provide a more detailed insight on the operational principle and construction of the device, and can be used as an introduction to e.g. to diffraction and polarization. In the following sections, short surveys of the different experimental modules will be given.

\section{AMPLITUDE MODULATION AND PROJECTION}

This experimental module is at an introductory level of difficulty. A white light source like an LED can be used, and the SLM is sandwiched between polarizers and lenses. The optical paths for imaging and illumination are, of course, different and should be understood by the students. One quantitative goal of this module is to determine the pixel size of the SLM from the amplification of the imaging setup.

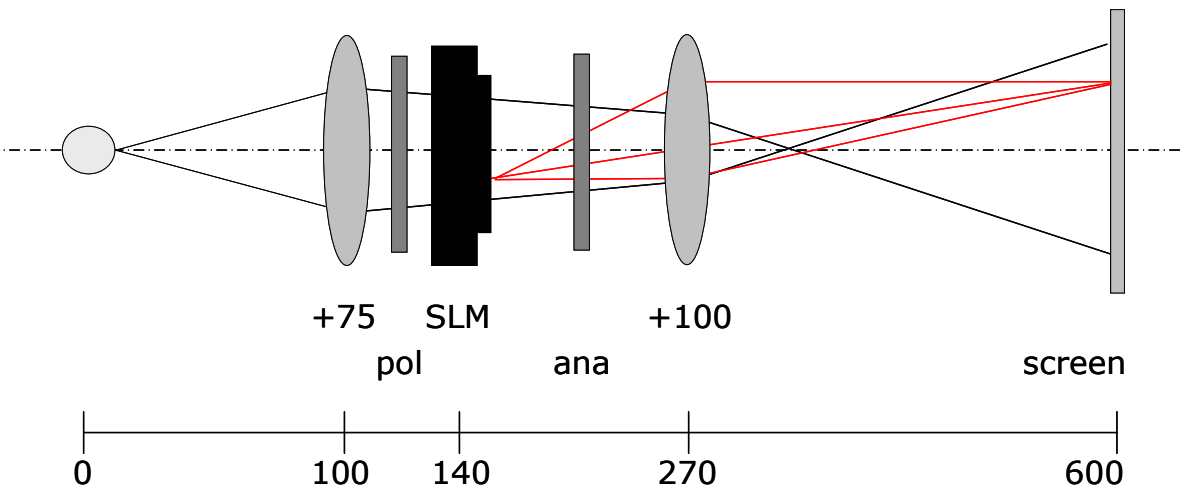

Figure 1: An example for a projector setup with the optical path for illumination (black) and the optical path for imaging (red)

The change of contrast of the obtained image can be determined as a function of the orientation direction of the polarizers, and the effect of the SLM on the polarization state of the light can be investigated for monochromatic light sources like red, green and blue LEDs. As can be seen in Figure 2, the orientation of the ellipse is rotated as a function of the addressed grey level.

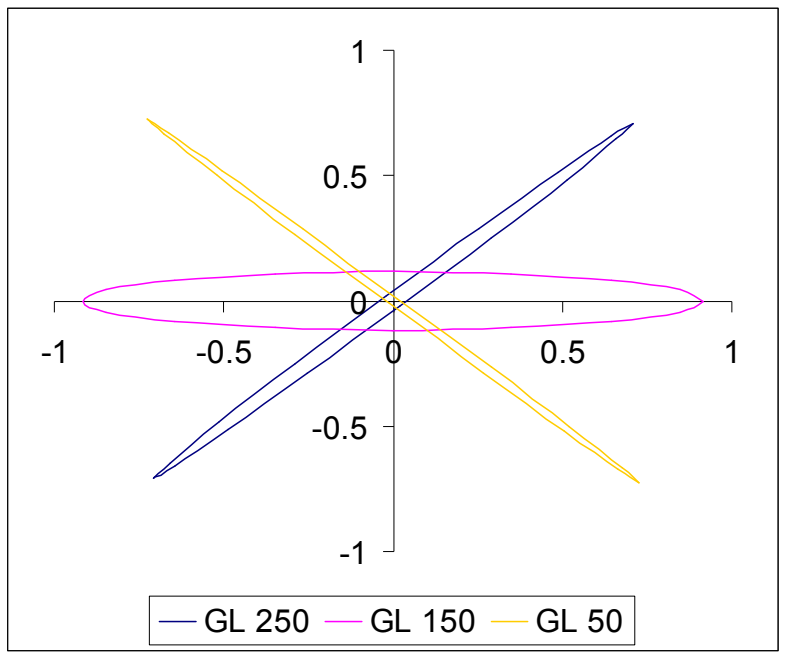

Figure 2: Rotated polarisation ellipses for different grey levels (GL's). 


\section{DETERMINATION OF JONES MATRIX REPRESENTATION AND TN-LC CELL PARAMETERS}

In this quite advanced module, the brief investigation of the change of polarization state done in the previous module is followed by a detailed investigation and analysis, which finally permit determination of the twist $\alpha$ of the LC cell and also its wavelength-dependent birefringence $\beta$. The experimental setup is simple, as can be seen in Figure 3.

The Jones matrix of the SLM can be written as a function

$$
\mathrm{W}_{\mathrm{TN}-\mathrm{LC}}^{\mathrm{fghj}}=e^{-\mathrm{i} \cdot \beta} \cdot\left(\begin{array}{cc}
f-\mathrm{i} \cdot g & h-\mathrm{i} \cdot j \\
-h-\mathrm{i} \cdot j & f+\mathrm{i} \cdot g
\end{array}\right)
$$

of the parameters $f, g, h$ und $j$, which are related to the physical parameters of the cell by

(2)

$$
\begin{aligned}
& f=\cos \gamma \cdot \cos \alpha+\frac{\alpha}{\gamma} \cdot \sin \gamma \cdot \sin \alpha \\
& h=\cos \gamma \cdot \sin \alpha-\frac{\alpha}{\gamma} \cdot \sin \gamma \cdot \cos \alpha \\
& g=\frac{\beta}{\gamma} \cdot \sin \gamma \cdot \cos (2 \psi-\alpha) \\
& j=\frac{\beta}{\gamma} \cdot \sin \gamma \cdot \sin (2 \psi-\alpha)
\end{aligned}
$$

where

$$
\gamma=\sqrt{\alpha^{2}+\beta^{2}}
$$

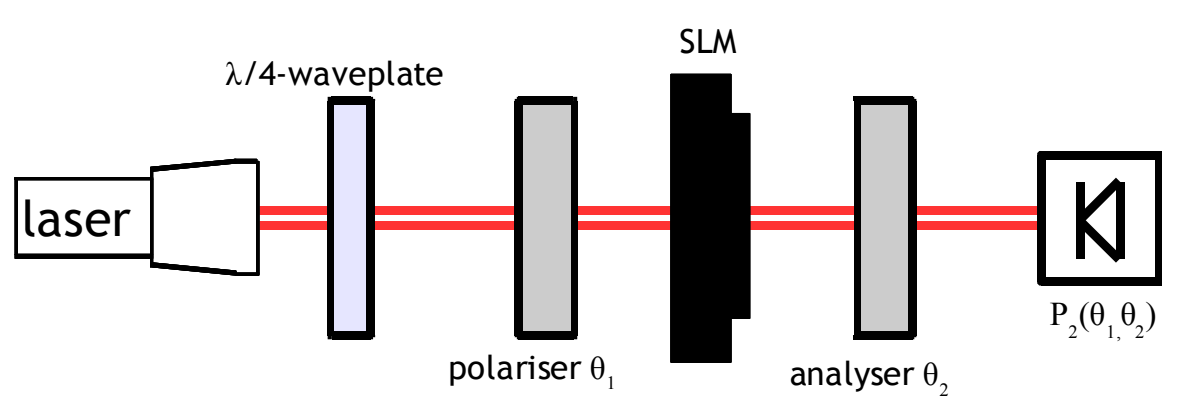

Figure 3: Transmission measurement through the LC display for determination of the Jones matrix components

It is rather straightforward to derive the transmission of the setup as a function of the polarizer orientation:

$$
\begin{aligned}
& \mathrm{T}^{+}\left(\theta_{1}\right)=\mathrm{T}\left(\theta_{1},+\theta_{1}\right)=f^{2}+\left(g \cdot \cos \left(2 \theta_{1}\right)+j \cdot \sin \left(2 \theta_{1}\right)\right)^{2} \\
& \mathrm{~T}^{-}\left(\theta_{1}\right)=\mathrm{T}\left(\theta_{1},-\theta_{1}\right)=g^{2}+\left(f \cdot \cos \left(2 \theta_{1}\right)+h \cdot \sin \left(2 \theta_{1}\right)\right)^{2}
\end{aligned}
$$


This measurement should preferably be repeated for at least three wavelengths (see Figure 4 for measured curves) in order to determine wavelength-dependent values for the parameters $f, g, h$ und $j$, and to derive the wavelength-dependent birefringence and the cell twist (which is independent of wavelength) from a numerical fit.

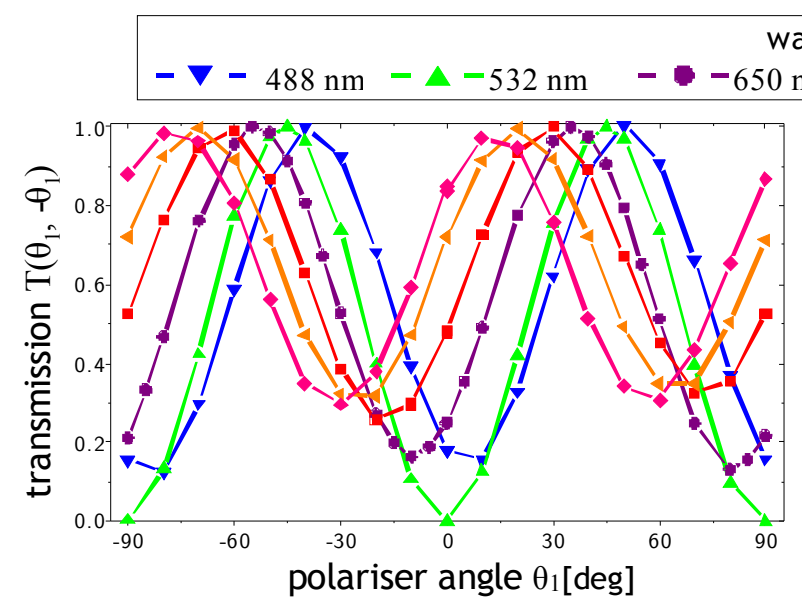

wavelength

$\mathrm{nm}-\square-780 \mathrm{~nm} \quad-4-980 \mathrm{~nm} \quad--1064 \mathrm{~nm}$

Figure 4: Transmission curves $\mathrm{T}^{-}\left(\theta_{1}\right)=\mathrm{T}\left(\theta_{1},-\theta_{1}\right)$ and $\mathrm{T}^{+}\left(\theta_{1}\right)=\mathrm{T}\left(\theta_{1},+\theta_{1}\right)$ for six different wavelengths

In the solution sets obtained for the different wavelengths, which are plotted in Figure 5, only one solution for the twist angle can be found for all six wavelengths: $\alpha=-90^{\circ}$. The birefringence values are given in table 1 . In summary, this module leads to an unambiguous determination of the physical cell parameters $\alpha$ and $\beta$

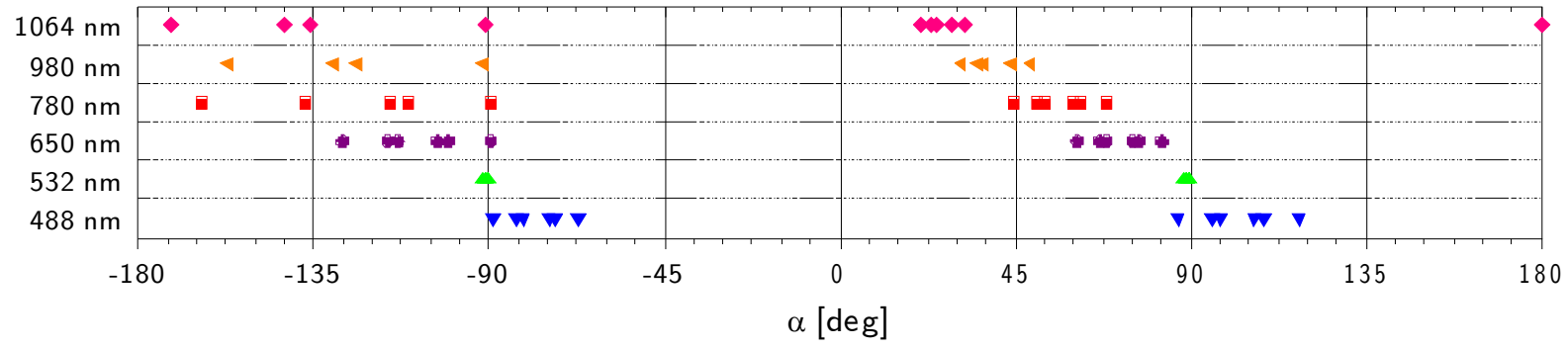

Figure 5: Solution sets obtained using a graphic-numerical analysis in the range $\left(-180^{\circ},+180^{\circ}\right)$ for the twist angle $\alpha$ at six different wavelengths with no voltage applied

\begin{tabular}{|c|c|c|c|}
\hline$\lambda[\mathrm{nm}]$ & $\alpha[\mathrm{deg}]$ & $\beta[\mathrm{rad}]$ & $\psi[\mathrm{deg}]$ \\
\hline 488 & -88.80 & 3.20 & -43.61 \\
\hline 532 & -90.03 & 2.69 & N/A \\
\hline 650 & -89.55 & 2.11 & -41.91 \\
\hline 780 & -89.76 & 1.75 & -42.19 \\
\hline 980 & -91.52 & 1.44 & -44.17 \\
\hline 1064 & -90.97 & 1.16 & -43.37 \\
\hline
\end{tabular}

Table 1: Solutions of the display parameters obtained from transmission curves with no voltage applied to the LC cell 


\section{INTERFEROMETRIC MEASUREMENT OF THE PHASE MODULATION}

The determination of the Jones matrix parameters can be done also for the case that voltages are applied to the SLM pixels by addressing grey-level images, but the related phase shift cannot be so easily determined from this method. Therefore in this module the phase shift is measured more directly by in interferometric fringe-shift technique. The phase shift $\Delta \Phi$ between two grey levels is given by the shift of the minima $\Delta y$ and the length of the period $g$ :

$$
\Delta \Phi=\frac{2 \pi}{g} \cdot \Delta y
$$

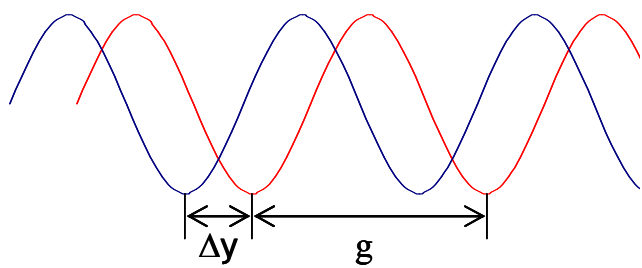

Figure 6: Shift of the interference minima

In the proposed setup, the fringe pattern produced by the two beams is imaged with moderate magnification to a CCD detector, as shown in Figure 7.

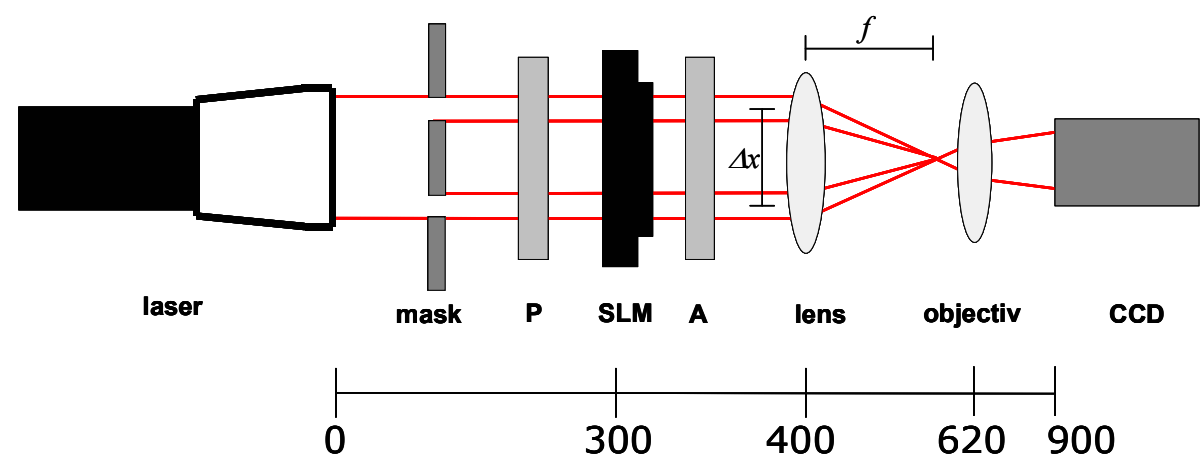

Figure 7: Two-beam interferometer to detect the phase shift

The variable phase modulation between the two beams is introduced by addressing half-screen images (see Figure 8). It is then rather straightforward to determine the relative phase shift from the results, as shown in Figure 9.
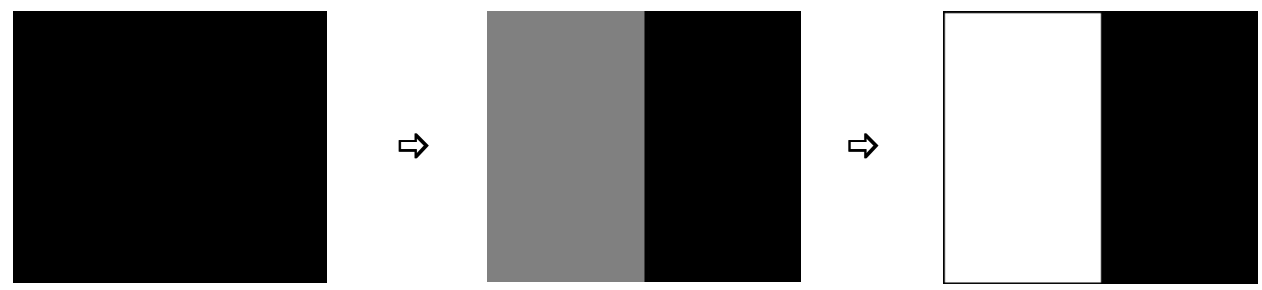

Figure 8: Examples for addressed half-screen graylevel images 


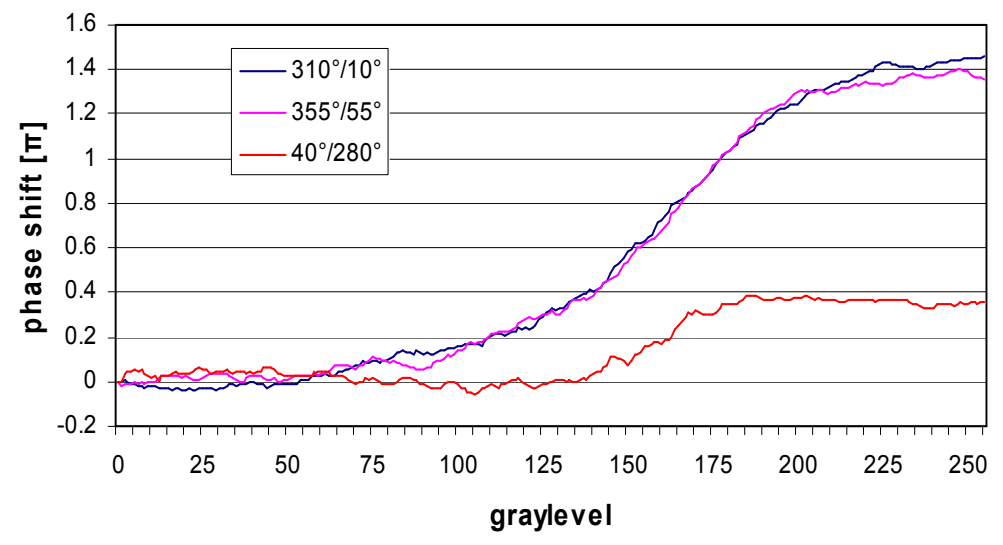

Figure 9: Phase shift of the display with different polariser/analyser configurations

\section{DIFFRACTION AT DYNAMICALLY ADDRESSED RONCHI GRATINGS}

In this module a different way to determine the phase shift is explored. By means of diffraction, a simple experimental setup (see Figure 10) is sufficient for this characterization.

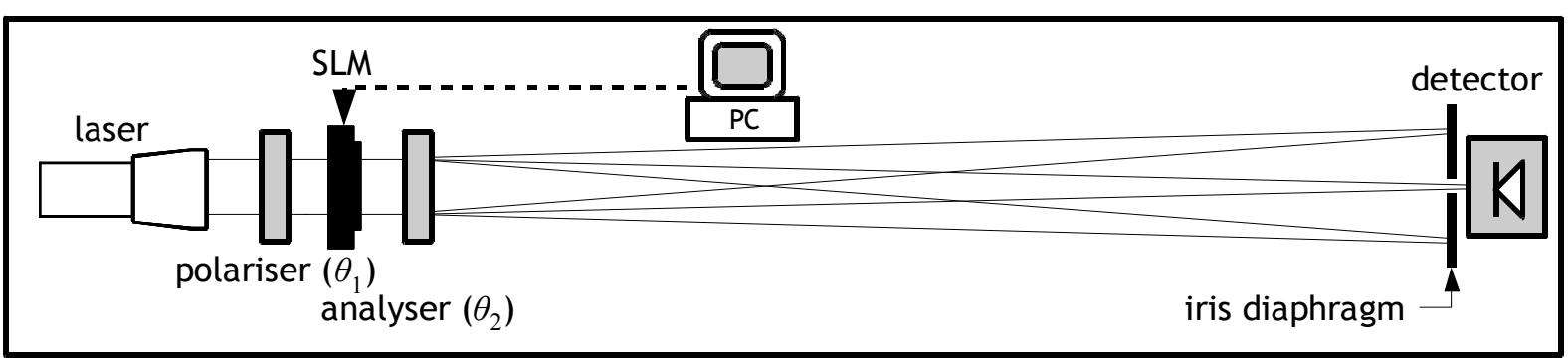

Figure 10: Experimental setup to measure the power transmission and the light power in diffraction orders

For measuring the power transmission, all SLM pixels are addressed uniformly with the same graylevel. From results shown in Figure 11 one can see that the dynamic range of the power transmission is heavily dependent on the orientation of the polarizers.

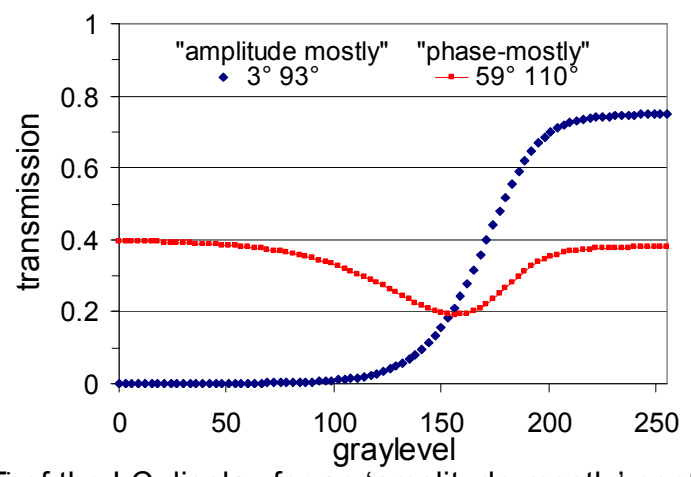

Figure 11: Power transmission $T_{i}$ of the LC display for an 'amplitude-mostly'-configuration $\left(\theta_{1}=3^{\circ}, \theta_{2}=93^{\circ}\right)$ and a 'phase-mostly'-configuration $\left(\theta_{1}=59^{\circ}, \theta_{2}=110^{\circ}\right)$ at $\lambda=650 \mathrm{~nm}$ 
For the determination of the phase modulation, Ronchi gratings with variable contrast are addressed, with a width of the ridges and grooves of e.g. 10 SLM pixels each. The diffraction efficiency of the $0^{\text {th }}$ order alone is already sufficient for the determination of the phase modulation. For a check of the accuracy, the phase modulation can also be derived from the $\pm 1^{\text {st }}$ orders. As can be seen from Figure 12, both ways of analysis deliver an almost identical result.
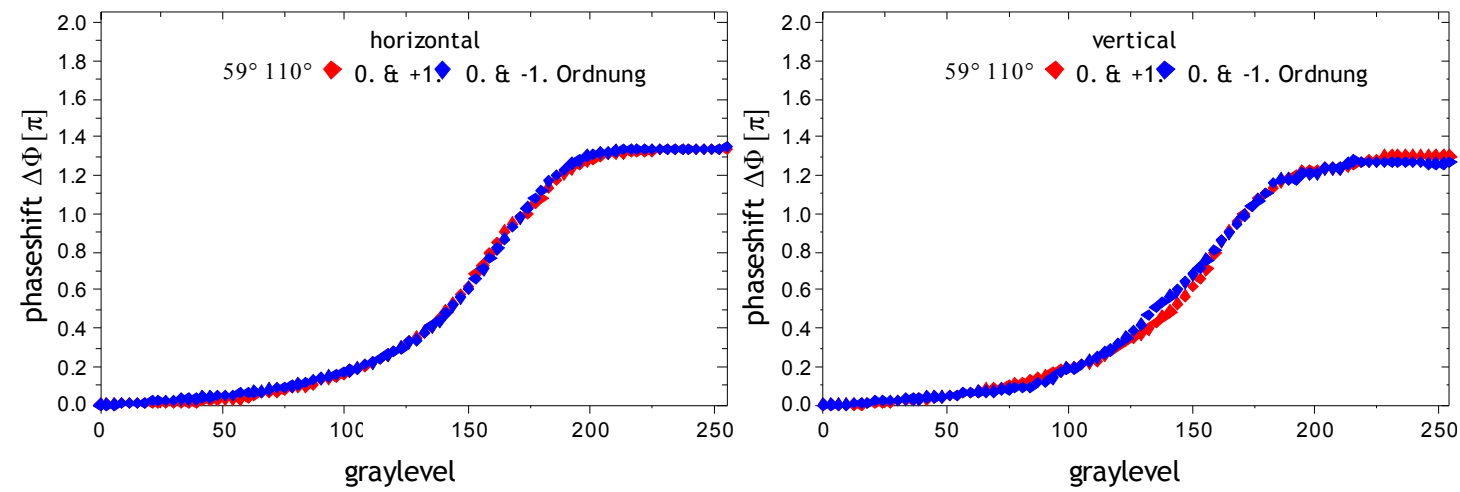

Figure 12: Calculated phase shift from transmission measurements in the 0 and $\pm 1^{\text {st }}$ diffraction orders in a 'phasemostly'-configuration $\left(\theta_{1}=59^{\circ}, \theta_{2}=110^{\circ}\right)$ with reference grey level 'black' at $\lambda=650 \mathrm{~nm}$

\section{LINEAR AND X-Y-SEPARABLE BINARY BEAM-SPLITTER GRATINGS}

The SLM can be used as an adaptive diffractive beam-splitter element. For an introduction to Fourier Optics and the Fourier series expansion of functions, linear gratings are analysed theoretically and experimentally in this experimental module. As shown in Figure 13, a lens can be used in front of the SLM to observe far-field diffraction at a finite distance.

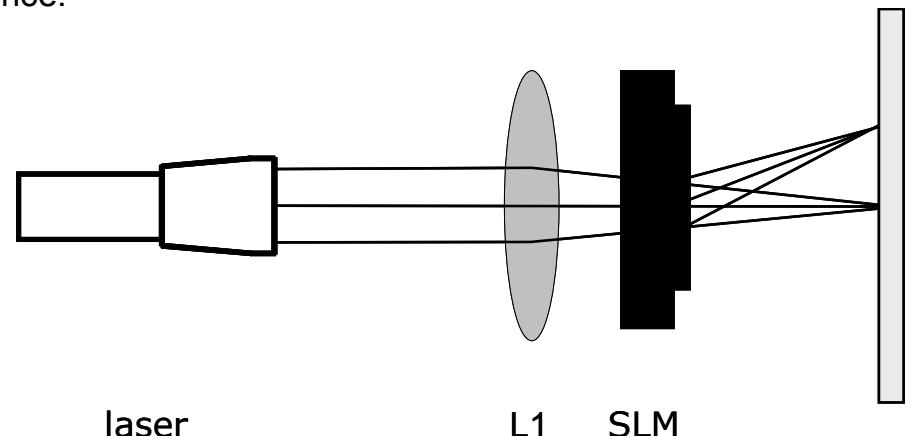

Figure 13: Fourier lens L1 in front of the light modulator

Even when the SLM is not addressed with a graylevel image, it already works as a diffraction grating due to its pixelized structure. The center-to-center distance in the 2D array of the LC cells can be determined by measurement of the diffraction angles of this 2D grating with a laser of known wavelength. Moreover, a measurement of the powers of these orders is interesting. In Figure 14, the powers measured along the horizontal and vertical directions are shown. The envelope of the diffraction efficiencies permits the estimation of the duty cycles $x_{1} / \mathrm{g}$ and $y_{1} / \mathrm{g}$ of the gratings in the two directions, because for a one-dimensional grating with any two transmission values $\tau_{1}$ and $\tau_{2}$ the diffraction efficiency is 


$$
\eta_{l}=\frac{\left|\tau_{2}-\tau_{1}\right|^{2}}{\pi^{2} \cdot l^{2}} \cdot \sin ^{2}\left(\pi l \frac{x_{1}}{g}\right),
$$

which means that for $x_{1}=g / k$ every diffraction order that satisfies $l=n k$ disappears. In the experimental results we have minima in about every sixth and every sixth order, respectively, so we have $x_{1} / \mathrm{g} \approx 1 / 6$ and $y_{1} / \mathrm{g} \approx 1 / 3$.
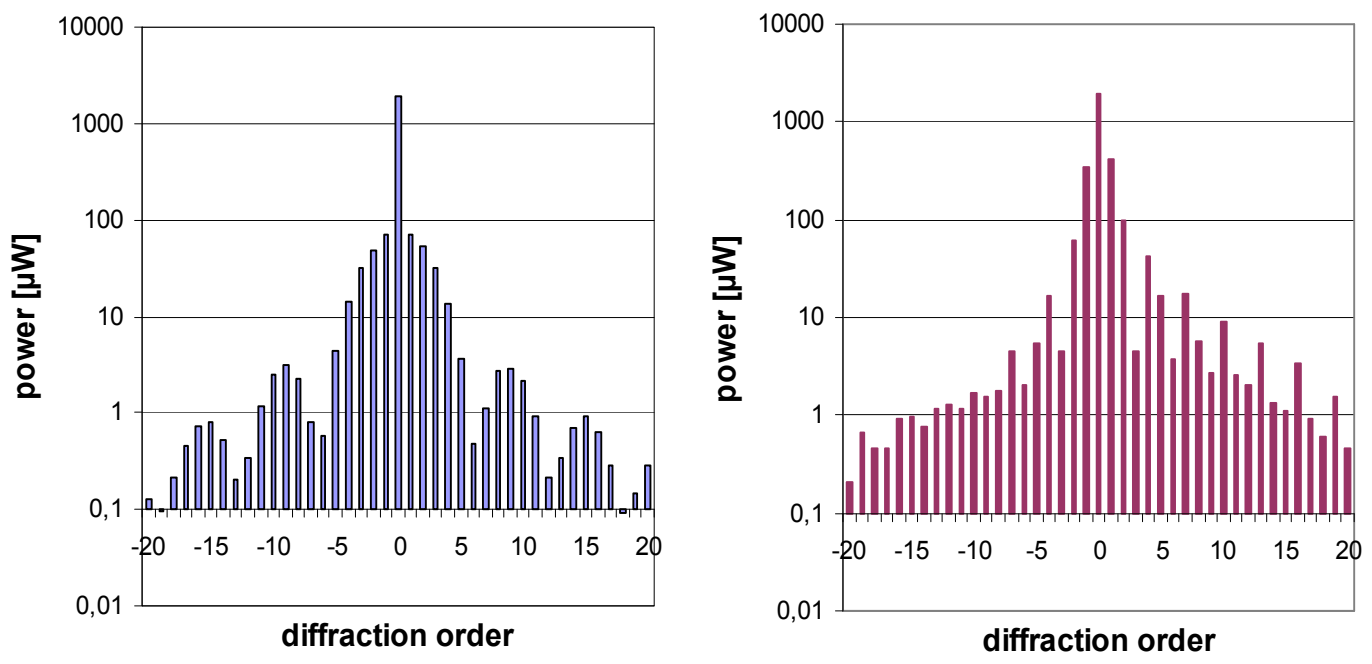

Figure 14: Measured power in horizontal (left) and vertical (right) diffraction orders at a non-addressed display

For the measurement of the diffraction orders of actively addressed phase gratings, the diffraction angles will be smaller because of the larger grating period which inevitably is given by a multiple of the pixel pitch. Therefore a setup as shown in Figure 15 is advisable, in which a diverging lens is used to enlarge the observed diffraction angles. For investigation of addressed binary optical elements with a phase shift of $\pi$, the polarizer and analyzer orientations and the two graylevels used for displaying the images should be chosen carefully from the results obtained in one of the two modules 4 and 5 described earlier.

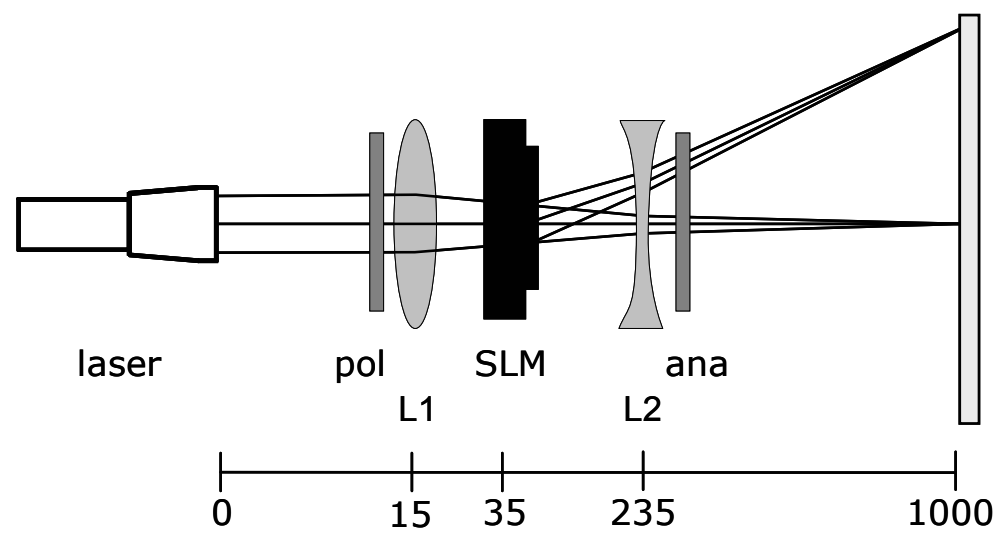

Figure 15: Setup for 'phase-mostly'-mode

Binary linear gratings are a particularly suitable subject for the comparison between theory and experiment. The theoretical analysis of is relatively easily done by analytic calculation of the Fourier series coefficients. The experiment is also easily doable, because only a small number of spot powers needs to be measured. When carefully set up, the agreement between theory and experiment can be very good (see table 2). 


\begin{tabular}{|c|c|c|c|}
\hline Measured value & power $[\mu \mathrm{W}]$ & diffraction efficiency [\%] & $\begin{array}{c}\text { theor. diffraction } \\
\text { efficiency [\%] }\end{array}$ \\
\hline 'Blank Screen' GL111 & 199 & $\mathrm{n} / \mathrm{a}$ & $\mathrm{n} / \mathrm{a}$ \\
\hline 'Blank Screen' GL203 & 204 & $\mathrm{n} / \mathrm{a}$ & $\mathrm{n} / \mathrm{a}$ \\
\hline 4/1/4/4/1/4-grating: $-3 r d$ order & 35.9 & 17.77 & 18.01 \\
\hline 4/1/4/4/1/4- grating: -1st order & 33.9 & 16.78 & 17.27 \\
\hline 4/1/4/4/1/4- grating: 0 order & 0.3 & 0.15 & 0 \\
\hline 4/1/4/4/1/4- grating: +1st order & 33.7 & 16.68 & 17.27 \\
\hline 4/1/4/4/1/4- grating: +3rd order & 36.0 & 17.82 & 18.01 \\
\hline
\end{tabular}

Table 2: Diffraction efficiency for an experimental setup delivering almost ideal binary phase-only modulation with a phase shift of $\pi$ for the selected polarizer and analyzer directions.

\section{COMPUTER GENERATED HOLOGRAMS AND ADAPTIVE LENSES}

The optical elements that can be represented by an SLM are not limited to gratings, and in this module more types of optical elements like kinoform holograms, lenses and prisms are investigated. The probably simplest of such elements is a binary Fresnel zone lens (FZL) which is made up from concentric rings. Addressing binary FZLs and determination of their focal length is another way for the students to determine the pixel size of the SLM, because the innermost radius $r$ of a binary zone lens is given by

$$
r=\sqrt{n \cdot \lambda \cdot f}
$$

and this radius is of course a multiple of the pixel size. This module is also dedicated to an short introduction into the computation of diffractive elements by numerical methods, and to acquirement and deepening of the understanding what the diffracted and undiffracted waves are when using an SLM, which in this situation is similar to a conventional hologram or a diffractive optical element (DOE).

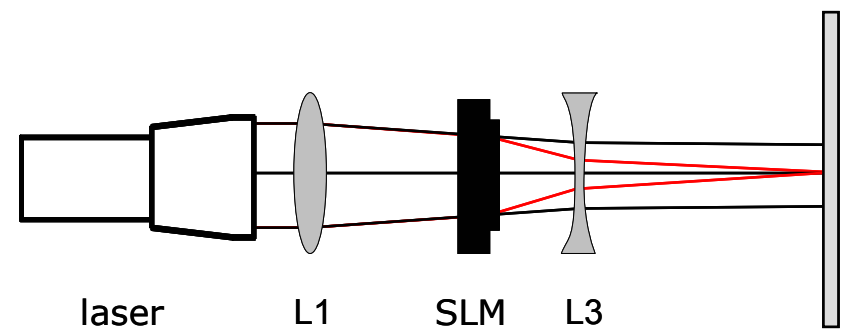

Figure 16: An added lens phase causes a focusing of the diffracted light (red). The undiffracted light is focused behind the screen (black).

The superposition of a spherical phase function representing a lens leads to a spatial shift of the far-field plane along the optical axis. As can be seen in Figure 17, the bright spot representing the undiffracted light wave in the center of the far-field observation plane is defocused in the new far field plane and appears as a background illumination. If in contrast a linear phase representing a prism is superimposed, the center of the diffraction pattern is shifted to the side. With software developed for the education kit, the superposition of these phase functions can be performed instantly, giving the students direct access to vary the settings. Quantitatively, the change of position of the far field diffraction pattern can be measured and related to the parameters of the linear or quadratic phase functions. 


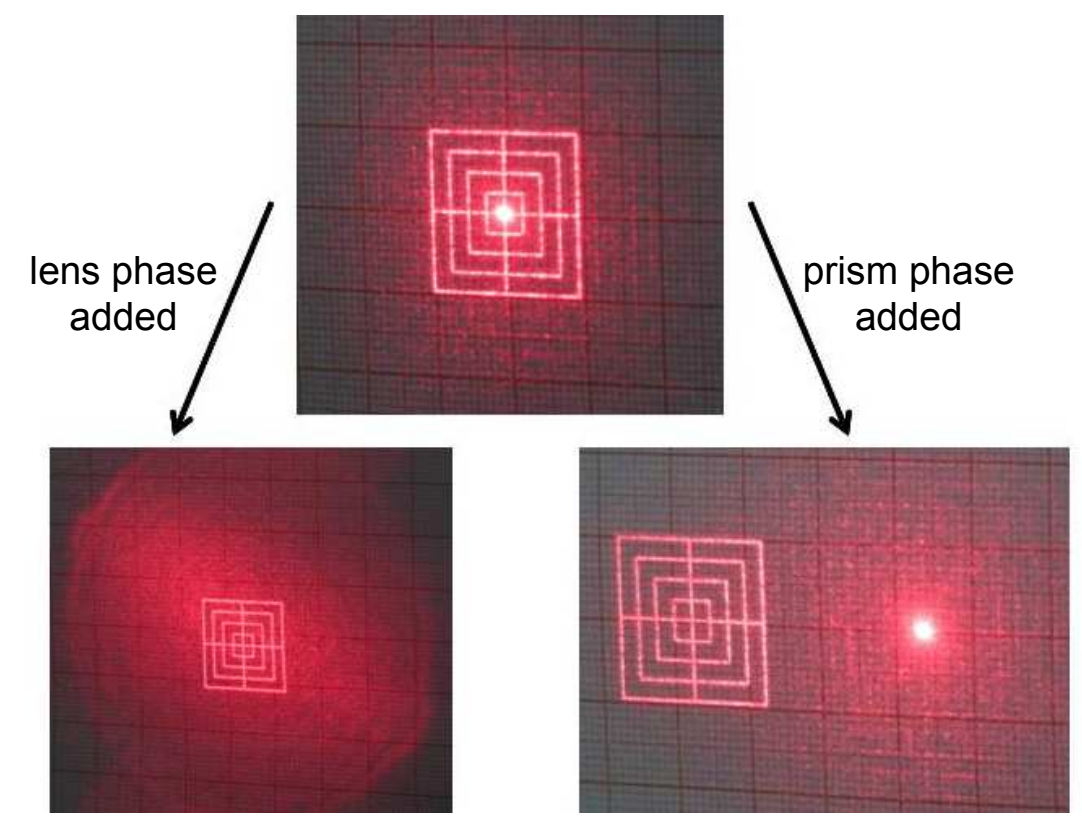

Figure 17: Top: focused zero order; Left: zero order defocused; Right: diffraction pattern shifted

\section{CONCLUSION AND OUTLOOK}

The existing modules already permit a flexible use in optics laboratories of university education, and the feedback we have obtained from the increasing number of users of the 'OptiXplorer' education kit is encouraging. We hope to develop the kit further and are interested in suggestions how to improve or extend it.

Of course it is possible to combine experiments from the different modules, for example in order to get a more particular focus on the characterization of the SLM or its application. The characterization of the SLM needs measurements from almost all six modules, including the cell birefringence and director plate orientations obtained from the Jones Matrix parameters analysis, the cell size and geometry from diffraction (with gratings or binary Fresnel Zone lenses) or from imaging in the projector setup, and the voltage-induced phase shift from a measurement with the fringe-shift interferometer or with the Ronchi grating diffraction experiment.

Also, one might also create an experimental module focusing more on the Fourier optical application of the SLM, by taking parts from the two related modules described here and adding more dedicated tasks to it. Further tasks which would fit into such development are the e.g. the set-up of an optical correlator, the investigation of the Talbot effect or a phase-contrast imaging set-up.

\section{ACKNOWLEDGEMENTS}

The theoretical introduction and the experimental tutorials have been created in cooperation with and incorporating feedback from several universities in Germany. We would like to thank our partners for their valuable contributions and suggestions for further improvement.

We would like to name the authors of the most substantial contributions:

- Prof. Dr. Ilja Rückmann, Dr. Tobias Voß - Universität Bremen

- PD Dr. Günther Wernicke, Humboldt-Universität zu Berlin

- Dipl.-Phys. Stephanie Quiram (AG Prof. H.J. Eichler), Technische Universität Berlin

- Dipl.-Ing. (FH) Sven Plöger (AG Prof. J. Eichler), Technische Fachhochschule Berlin 